\title{
$\mathrm{BMJ}$
}

\section{Association between general and central adiposity in childhood, and change in these, with cardiovascular risk factors in adolescence: prospective cohort study}

\author{
Debbie A Lawlor, professor of epidemiology, ${ }^{1,2}$ Li Benfield, research associate, ${ }^{1,2}$ Jennifer Logue, clinical \\ lecturer in metabolic medicine, ${ }^{3}$ Kate Tilling, reader in statistics, ${ }^{2}$ Laura D Howe, research associate, ${ }^{1,2}$ Abigail \\ Fraser, research fellow, ${ }^{1,2}$ Lynne Cherry, research staff, ${ }^{3}$ Pauline Watt, research staff,, Andrew R Ness, \\ professor of epidemiology, ${ }^{4}$ George Davey Smith, professor of epidemiology, ${ }^{1,2}$ Naveed Sattar, professor of \\ metabolic medicine ${ }^{3}$
}

\section{MRC Centre for Causal Analyses in Translational Epidemiology, University of Bristol, Bristol BS8 2BN, UK \\ ${ }^{2}$ School of Social and Community Medicine, University of Bristol, Bristol \\ Institute of Cardiovascular and Medical Sciences, BHF Glasgow Cardiovascular Research Centre, University of Glasgow, Glasgow ${ }^{4}$ Department of Oral and Dental Science, University of Bristol, Bristol}

Correspondence to: D Lawlor d.a.lawlor@bristol.ac.uk

Cite this as: $B M J$ 2010;341:c6224 doi:10.1136/bmi.c6224

\section{ABSTRACT}

Objectives To examine the prospective associations between body mass index (BMI), waist circumference, and fat mass in childhood and cardiovascular risk factors at age 15-16.

Design Prospective cohort study.

Setting Avon Longitudinal Study of Parents and Children. Participants 5235 children aged 9-12 at start of study.

Main exposures BMI, waist circumference, and fat mass determined by dual energy $\mathrm{x}$ ray absorptiometry, assessed at age 9-12 and at age 15-16.

Main outcome measures Systolic and diastolic blood pressure and concentrations of fasting glucose, insulin, triglycerides, low density lipoprotein cholesterol, and high density lipoprotein cholesterol assessed at age 1516.

Results In girls a 1 SD greater BMI at age 9-12 was associated with cardiovascular risk factors at age 15-16 in fully adjusted models: odds ratio 1.23 (95\% confidence interval 1.10 to 1.38 ) for high systolic blood pressure ( $\geq 130 \mathrm{~mm} \mathrm{Hg}$ ); 1.19 (1.03 to 1.38) for high concentration of low density lipoprotein cholesterol ( $\geq 2.79 \mathrm{mmol} / \mathrm{l})$; 1.43 (1.06 to 1.92$)$ for high concentration of triglycerides ( $\geq 1.7 \mathrm{mmol} / \mathrm{l}) ; 1.25$ (1.08 to 1.46 ) for low concentration of high density lipoprotein cholesterol ( $11.03 \mathrm{mmol} / \mathrm{l})$; and 1.45 (1.22 to 1.73) for high concentration of insulin ( $\geq 16.95 \mathrm{IU} / \mathrm{l})$. Equivalent results in boys were 1.24 (1.13 to 1.37 ) for systolic blood pressure; 1.30 (1.07 to 1.59) for low density lipoprotein cholesterol; 1.96 (1.51 to 2.55) for triglycerides; 1.39 (1.22 to 1.57 ) for high density lipoprotein cholesterol, and 1.84 (1.56 to 2.17) for insulin. BMI was associated with high fasting glucose ( $\geq 5.6 \mathrm{mmol} / \mathrm{l}$ ) only in boys $(1.18,1.03$ to 1.36 ). With these binary outcomes there was statistical evidence that associations differed between girls and boys for fasting glucose $(P=0.03)$ and insulin ( $P<0.001)$. When risk factors were examined as continuous outcomes there was evidence for stronger associations of BMI with more adverse levels in boys than girls for fasting insulin, glucose, and triglyceride concentrations (all interaction
$\mathrm{P} \leq 0.03)$. BMI, waist circumference, and fat mass were all strongly correlated with each other $(r=0.89-0.94)$, and associations of the three with cardiovascular outcomes were of similar magnitude with statistical evidence of consistency in associations (all P>0.2 for heterogeneity). When waist circumference or fat mass or both were added to models including BMI they did not increase the variation in cardiovascular risk factors already explained by BMI and confounders alone. Girls who were overweight/obese at age $9-12$ but were normal weight by 15-16 had similar odds of adverse levels of risk factors to those who were normal weight at both ages. In boys odds of high systolic blood pressure, high concentrations of triglycerides and insulin, and low concentrations of high density lipoprotein cholesterol remained higher in this group compared with those who were normal weight at both ages but were lower than in those who remained overweight/obese at both ages.

Conclusions Measurements of waist circumference or directly assessed fat mass in childhood do not seem to be associated with cardiovascular risk factors in adolescence any more strongly than BMI. Girls who favourably alter their overweight status between childhood and adolescence have cardiovascular risk profiles broadly similar to those who were normal weight at both time points, but boys who change from overweight to normal show risk factor profiles intermediate between those seen in boys who are normal weight at both ages or overweight at both ages.

\section{INTRODUCTION}

Higher body mass index (BMI) in childhood or adolescence is associated with an increased risk of cardiovascular disease in later life, with this association being linear across most of the BMI distribution. ${ }^{1-3}$ Childhood BMI is also associated with cardiovascular risk factors, carotid intima media thickness, and left ventricular mass in early or mid-adulthood..$^{4-8}$ Several cross sectional studies have shown associations between measurements of childhood adiposity, most 
commonly BMI, and cardiovascular risk factors. ${ }^{9-12}$ These associations, however, have rarely been explored prospectively, and previous studies have not compared different measurements of adiposity and its distribution. BMI has been criticised as an inadequate measure of adiposity, particularly in children, in whom annual increases in BMI can reflect increases in lean mass more so than fat mass, ${ }^{1314}$ but it is unknown whether more direct measurement of fat, or its distribution, can predict cardiovascular risk factors more strongly than BMI in children. ${ }^{12}$

We determined the association between BMI, waist circumference, and directly assessed fat mass assessed at age 9-12, and the change in these between 9-12 and 15-16, with cardiovascular risk factors assessed at age 15-16. We compared the magnitudes of association between the three measurements of adiposity to determine whether either a direct measure of fat mass or a measure of central adiposity distribution (waist circumference) was more strongly associated than BMI with cardiovascular risk factors.

\section{METHODS}

We used data from the Avon Longitudinal Study of Parents and Children (ALSPAC), a longitudinal population based birth cohort that recruited 14541 pregnant women with expected delivery dates from 1 April 1991 to 31 December 1992 (www.alspac.bris. ac.uk). ${ }^{15}$ Since age 7 surviving offspring have been invited to regular follow-up clinics. In the current analysis we examined data from three clinics conducted when the children were aged 9-10, 11-12, and 15-16.

Each clinic used the same protocols for assessing adiposity. Current age of the child was recorded in months. Weight and height were measured with the child in light clothing and without shoes. Weight was measured to the nearest $0.1 \mathrm{~kg}$ with Tanita scales and height to the nearest $0.1 \mathrm{~cm}$ with a Harpenden stadiometer. A flexible tape was used to measure waist circumference to the nearest $1 \mathrm{~mm}$ at the mid-point between the lower ribs and the pelvic bone. A Lunar Prodigy narrow fan beam densitometer was used to perform whole body dual energy x ray absorptiometry to measure lean and fat mass. For our main analyses we used sex, age (in month categories), and standard deviation scores (z scores) of BMI, waist circumference, and fat mass to compare the magnitudes of their associations. As in a previous study ${ }^{16}$ we used baseline adiposity data for these analyses by combining data from either the 9-10 or 11-12 clinic, using the 9-10 year assessment for all who had these measurements and the 11-12 year assessments for those who did not.

Blood pressure (at all clinics) was measured with a Dinamap 9301 Vital Signs Monitor (Morton Medical, London) with the correct cuff size. Two readings of systolic and diastolic blood pressure were recorded, with the child at rest and their arm supported, and we used the mean of the two measures.

At the 15-16 year clinic participants were asked to fast overnight for those attending in the morning or for a minimum of six hours for those attending after lunch. Blood samples were immediately spun and frozen at $-80^{\circ} \mathrm{C}$. Measurements were assayed three to nine months after samples were taken, with no previous freeze-thaw cycles. Plasma lipid concentrations (total cholesterol, triglycerides, and high density lipoprotein cholesterol) were measured by modification of the standard Lipid Research Clinics Protocol by using enzymatic reagents for lipid determination. Low density lipoprotein cholesterol concentration was determined from these with the Friedwald equation (low density lipoprotein cholesterol=total cholesterol -(high density lipoprotein cholesterol+triglycerides $\times 0.45)$ ). Insulin was measured with an enzyme linked immunosorbent assay (ELISA) (Mercodia, Uppsala, Sweden) that does not cross-react with proinsulin, and plasma glucose was measured with an automated assay.

Potential confounding factors considered included maternal age at birth of the child, parity, household occupational social class, parental education, birth weight, height, gestational age, parental BMI, age of the child (at the time of assessment of BMI, waist circumference, or fat mass), and pubertal status (assessed by parental report at baseline with Tanner questionnaires and whether girls had gone through menarche and by self report at outcome with the same instruments). All results are presented separately for girls and boys and therefore sex is not considered a confounder. Full details of how these variables were assessed are provided in the supplementary material on bmj.com.

\section{Statistical analysis}

All analyses were conducted in Stata/MP 11.0. Correlations between all anthropometric measurements were assessed with Pearson's correlation coefficient by using the anthropometric measurements on their original scale (that is, not as SD scores). Repeating these with age and sex standardised $\mathrm{z}$ scores did not change the results nor did additional adjustment (partial correlation coefficients) for age and sex (data available from authors on request).

Because there was evidence of sex differences in some of the main associations, we have presented all regression results separately by sex and $P$ values for the null hypothesis of no difference in association between girls and boys (exposure-sex interaction).

\section{Dealing with missing data}

Over the study, 7725, 7159, and 5509 participants attended the 9-10 year, 11-12 year, and 15-16 year clinics, respectively. Our eligible study sample included any child who attended either the 9-10 year or 11-12 year clinic ( $\mathrm{n}=8362)$ and also attended the $15-16$ year clinic; 5235 individuals fulfilled these criteria.

To increase efficiency and minimise selection bias we used multivariate multiple imputation to impute missing variables for participants considered to be eligible (that is, those attending one or other of the 9-10 or 11-12 year assessment and also the 15-16 year 
assessment), including all exposures, covariables, outcomes, and potential predictors of missing data in the imputation equations. ${ }^{17}$ Full details of this procedure are provided in the supplementary material on bmj.com. Tables B (girls) and C (boys) on bmj.com compare the distributions of imputed variables in the imputed datasets and the observed data (with no imputation) and show that distributions in imputed datasets were similar to those observed, providing some evidence that the missing data were missing at random (an assumption of this imputation procedure). We also repeated all analyses including only those with complete data on all variables used in any analysesthat is, without imputation $(\mathrm{n}=1785)$.

\section{Prospective associations of adiposity with cardiovascular risk factors}

To provide clinical insights for our main analyses we examined associations with each risk factor as a binary outcome, comparing those who had levels above (or below for high density lipoprotein cholesterol) a threshold that indicates increased risk in this age group. The international diabetes federation provides thresholds for defining children/adolescents at risk of future cardiovascular disease for triglycerides, high density lipoprotein cholesterol, glucose, and blood pressure in the age group $10-<16$ and states that adult thresholds should be used in those aged $\geq 16 .{ }^{18}$ The mean (SD) age of participants at assessment of risk factors in our study was 15.5 (0.3), and $91 \%$ were under 16. We therefore applied the international diabetes federation thresholds to all participants, and in a sensitivity analysis repeated analyses with those aged $\geq 16$ $(9 \%)$ assigned high risk if they had values above adult thresholds. The results from this sensitivity analysis (including the prevalence to 1 decimal place of those at risk) did not differ from the results of analyses that used the childhood/adolescent thresholds for all participants, and we present only results using the child/ adolescent thresholds. For low density lipoprotein cholesterol and insulin the international diabetes federation does not provide childhood/adolescent thresholds, and even in adults there is no agreed threshold of hyperinsulinaemia. For these two risk factors we used those who had values equal to or over the 90 th centile in our cohort to indicate being at risk. Thresholds used to indicate future increased risk of cardiovascular disease for each risk factor were $\geq 130 \mathrm{~mm} \mathrm{Hg}$ for systolic blood pressure, $\geq 85 \mathrm{~mm} \mathrm{Hg}$ for diastolic blood pressure, $\geq 1.7 \mathrm{mmol} / 1$ for triglycerides, $<1.03 \mathrm{mmol} / 1$ for high density lipoprotein cholesterol; $\geq 2.79 \mathrm{mmol} / \mathrm{l}$ for low density lipoprotein cholesterol, $\geq 5.6 \mathrm{mmol} / 1$ for glucose, and $\geq 16.95 \mathrm{IU} / 1$ for insulin.

We also examined associations with risk factors as continuous outcomes. Fasting insulin and triglyceride concentrations were positively skewed, and therefore we used log values in regression analyses; distributions of insulin and triglyceride and of the residuals in the regression models were normal with these transformations. The results from the regression analyses with $\log$ insulin and triglyceride concentration were exponentiated to give ratios of geometric means per $\mathrm{SD}$ (z scores) change in adiposity. A ratio of geometric means is a proportionate difference with a null value of 1. For example, a ratio of geometric means of $1.10 \mathrm{sug}$ gests a $10 \%$ difference between exposure groups or levels in the outcome.

We used multivariable logistic regression (for binary outcomes) and linear regression (for the risk factors as continuous outcomes) to examine associations of each of the adiposity measurements with the cardiovascular risk factors. We adjusted for age in months at the time of assessment of adiposity in all models. We also adjusted for height and height ${ }^{2}$ for all adiposity markers because at age 9-12 all of these measurements, including BMI, were positively associated with height. The fully adjusted model additionally controlled for maternal age at birth of the child, parity, household occupational social class, parental education, birth weight, gestational age, parental BMI, and pubertal status.

\section{Examining linear $v$ threshold associations}

We used two methods to examine whether associations were linear across the distribution of adiposity risk factors or driven primarily by increased risk only in those at the upper end of the distribution of adiposity. Firstly, we split the exposure into fifths, plotted mean levels of risk factors across these fifths, and looked at these graphs to see if there was any clear evidence of a threshold effect. We ran regression models with these fifths as four indicator variables (non-linear) and compared this model with one in which the fifths were included as a single variable (a score from 1 to 5 ; linear model across fifths). We used a likelihood ratio test for the comparison between these two models. Secondly, we repeated multivariable analyses in children defined as normal weight (that is, excluding overweight/obese based on sex and age specific cut points for BMI from the International Obesity Task Force (IOTF) ${ }^{19}$ (see table D on bmj.com for the cut points used).

Change in adiposity and risk factors for cardiovascular disease We examined the association of change in adiposity between 9-12 years and 15-16 years and cardiovascular risk factors assessed at 15-16 years in two ways. Firstly, we used a continuous variable of the adiposity $\mathrm{z}$ scores at age 15-16 minus the $\mathrm{z}$ scores at age 9-12. We included that as the main exposure variable in regression models and additionally adjusted for baseline (9-12 years) adiposity. Secondly, we examined associations of change in categories of overweight/obesity status between age 9-12 and 15-16 with cardiovascular risk factors. For BMI overweight/obesity was defined according to criteria from the International Obesity Task Force ${ }^{19}$; for waist circumference, central overweight/obesity was defined as being at ot above the 90 th centile ${ }^{18}$ with age and sex specific centile curves derived for British children/adolescents ${ }^{20}$; for fat mass (in the absence of any guidance or national age and sex specific growth curves) we defined those above the 90 th centile for age and sex in our cohort as being overweight/obese. 
Heterogeneity in associations between different measurements of adiposity

Because the estimates from models with each of the three adiposity measurements are not from independent studies (they are all from the same cohort) it would not be appropriate to use standard tests of heterogeneity (such as $\mathrm{I}^{2}$ or a $\mathrm{Q}$ statistic) used in meta-analyses. Therefore, we used 1000 bootstrap replications ${ }^{21}$ to estimate standard errors of differences between log odds (for binary outcomes) and between means (for continuously measured outcomes) between models (for example, between the associations of BMI with systolic blood pressure and waist circumference and systolic blood pressure). We calculated $\mathrm{P}$ values from these standard errors based on a normal approximation for the sampling distribution of the log odds ratios/mean differences, after checking the bootstrap distributions were close to normal using normal probability plots (all were). These $\mathrm{P}$ values provide a test of heterogeneity between the estimates with different measurements of adiposity.

Our main interest here was to determine whether the magnitude of association between BMI and cardiovascular risk was as strong as that between more directly assessed fat mass and centrally distributed fat and these outcomes. The appropriate way to address this is to examine associations with each exposure separately and then compare the resulting regression coefficients as described above. If the magnitudes of association are similar this suggests that use of BMI in routine clinical practice and public health surveillance is valuable and will not importantly underestimate risk. Two related questions are whether adding other measurements of adiposity to a model with BMI already included will increase the ability to predict risk compared with BMI alone and whether when all three are included in a model together there is evidence that they are all independently (of each other) related to outcomes. We have not addressed these questions in great detail here as we feel to do so requires considerably more space. Furthermore, the strong correlations between all three adiposity measurements (see below) makes inclusion of two or all three of them in the same regression model potentially problematic because of colinearity. We have done one final set of analyses in which we compare the percentage of variation in the outcome explained by exposure variables in combination from a series of regression models (using $100 \times \mathrm{R}^{2}$ ) with outcomes as continuous variables. The series of regression models that we compared were: all potential confounders but none of the adiposity measures; all potential confounders and BMI; all potential confounders, BMI, and waist circumference; all potential confounders, BMI, and fat mass; and all potential confounders, BMI, waist circumference, and fat mass. We also determined the variance inflation factor (a measure of colinearity) for each adiposity measure in all but the first model; variance inflation factors over 10 are considered to introduce problems of colinearity. ${ }^{22}$

\section{RESULTS}

The characteristics of the cohort are shown in table E on bmj.com. According to International Obesity Task Force (IOTF) criteria, at baseline (age 9-12) 18.5\% (965) of the participants were overweight and 4.5\% (234) were obese. The prevalence of cardiovascular risk factors at age 15-16 varied between $2.9 \%$ for high diastolic blood pressure (144) and triglyceride concentrations (95) to $28.8 \%$ (1421) for high systolic blood pressure. Table F on bmj.com shows the correlations between parental and childhood anthropometric measurements; correlations were similar in girls and boys (all $\mathrm{P}>0.5$ for interaction) and therefore results were combined. At each of the three ages all three of the measurements of adiposity were strongly correlated with each other $(r=0.89-0.94)$, and each measurement of adiposity was strongly positively correlated with the same measurement at different ages $(r=0.63-0.93)$.

Table G on bmj.com shows the cross sectional associations of BMI, waist circumference, and fat mass assessed at age 15-16 with the cardiovascular risk factors treated as binary outcomes, and table $\mathrm{H}$ on bmj.com shows the same cross sectional associations with outcomes as continuous variables, for all eligible participants with missing data imputed. See the appendix on bmj.com for a full discussion of these results.

Table 1 shows the prospective associations of childhood (9-12 years) BMI, waist circumference, and fat mass with cardiovascular risk factors (as binary variables) at age 15-16 years. In girls and boys all three adiposity measures were associated with increased odds of adverse levels of systolic blood pressure and adverse concentrations of fasting low density lipoprotein cholesterol, triglycerides, high density lipoprotein cholesterol, and insulin. In boys only they were also associated with increased odds of high glucose concentration. Adiposity measures were not associated with high diastolic blood pressure in either sex. Adjustment for potential confounding factors did not substantively alter associations. The pattern of associations were similar with outcomes on the continuous scale (table 2). With outcomes assessed as binary or continuous measurements there was statistical evidence for sex interactions (stronger associations in boys than in girls) for fasting insulin, and, additionally, when assessed on the continuous scale there was evidence of a stronger association in boys than girls for fasting glucose and triglycerides for all three measures of adiposity. There was statistical evidence that associations were consistent with each other whether BMI, waist circumference, or fat mass was used (all $\mathrm{P} \geq 0.2$ for heterogeneity from bootstrap analyses).

When we restricted analysis to children who were normal weight, all associations were similar to those presented (results available from authors), suggesting that they were not driven solely by a threshold effect of increased risk in those at the upper end of the distribution. When associations existed they were linear across the distribution of values (all $\mathrm{P}<0.001$ for linear trend), with no evidence of threshold effects with marked 
Table 1|Prospective associations between BMI, waist, and fat mass (assessed at age 9-12) and cardiovascular risk factors as binary outcomes at age 15-16 in all eligible participants with missing data imputed by multivariate multiple imputation $(n=5235)$. Figures are odds ratios of outcome per 1SD ( $z$ score) of exposure ( $95 \%$ confidence interval)

\begin{tabular}{|c|c|c|c|c|c|}
\hline \multirow[b]{2}{*}{ Exposure and outcome* } & \multicolumn{2}{|c|}{ Girls $(n=2747)$} & \multicolumn{2}{|c|}{ Boys $(n=2488)$} & \multirow{2}{*}{$\begin{array}{l}\text { P value for sex } \\
\text { interaction§ }\end{array}$} \\
\hline & Model 1† & Model $2 \ddagger$ & Model 1† & Model $2 \ddagger$ & \\
\hline \multicolumn{6}{|l|}{ BMI } \\
\hline High systolic BP & 1.24 (1.13 to 1.37$)$ & 1.23 (1.10 to 1.38$)$ & $1.26(1.15$ to 1.37$)$ & 1.24 (1.13 to 1.37$)$ & 0.91 \\
\hline High diastolic BP & 1.05 (0.79 to 1.39$)$ & 1.11 (0.81 to 1.52$)$ & $1.01(0.82$ to 1.25$)$ & $1.06(0.84$ to 1.35$)$ & 0.37 \\
\hline High LDLC & 1.24 (1.09 to 1.40$)$ & 1.19 (1.03 to 1.38$)$ & 1.33 (1.11 to 1.59$)$ & 1.30 (1.07 to 1.59$)$ & 0.10 \\
\hline High triglycerides & 1.35 (1.02 to 1.78$)$ & 1.43 (1.06 to 1.92$)$ & $1.93(1.54$ to 2.41$)$ & 1.96 (1.51 to 2.55$)$ & 0.49 \\
\hline Low HDLc & 1.37 (1.20 to 1.57$)$ & 1.25 (1.08 to 1.46$)$ & $1.43(1.27$ to 1.61$)$ & 1.39 (1.22 to 1.57$)$ & 0.24 \\
\hline High glucose & 1.06 (0.87 to 1.29$)$ & $1.03(0.84$ to 1.27$)$ & $1.22(1.07$ to 1.38$)$ & 1.18 (1.03 to 1.36$)$ & 0.03 \\
\hline High insulin & 1.53 (1.31 to 1.79$)$ & $1.45(1.22$ to 1.73$)$ & 1.88 (1.63 to 2.16$)$ & 1.84 (1.56 to 2.17$)$ & $<0.001$ \\
\hline \multicolumn{6}{|l|}{ Waist circumference } \\
\hline High systolic BP & 1.21 (1.09 to 1.35$)$ & 1.18 (1.05 to 1.33$)$ & $1.24(1.12$ to 1.36$)$ & 1.20 (1.08 to 1.33$)$ & 0.85 \\
\hline High diastolic BP & $1.08(0.80$ to 1.46$)$ & 1.14 (0.82 to 1.58$)$ & $0.91(0.71$ to 1.16$)$ & $0.93(0.71$ to 1.22$)$ & 0.31 \\
\hline High LDLC & 1.28 (1.11 to 1.47$)$ & $1.23(1.05$ to 1.43$)$ & $1.29(1.08$ to 1.54$)$ & 1.29 (1.03 to 1.54$)$ & 0.10 \\
\hline High triglycerides & $1.36(1.00$ to 1.87$)$ & 1.42 (1.01 to 1.99$)$ & 1.96 (1.57 to 2.46$)$ & 1.97 (1.50 to 2.59$)$ & 0.99 \\
\hline Low HDLc & 1.41 (1.23 to 1.62$)$ & $1.29(1.12$ to 1.49$)$ & $1.45(1.30$ to 1.62$)$ & 1.40 (1.25 to 1.57$)$ & 1.00 \\
\hline High glucose & 1.09 (0.87 to 1.33$)$ & 1.06 (0.84 to 1.32$)$ & 1.18 (1.03 to 1.35$)$ & 1.14 (0.99 to 1.32$)$ & 0.003 \\
\hline High insulin & 1.58 (1.32 to 1.88$)$ & 1.47 (1.20 to 1.80$)$ & 1.89 (1.64 to 2.18$)$ & 1.84 (1.56 to 2.17 ) & $<0.001$ \\
\hline \multicolumn{6}{|l|}{ Fat mass } \\
\hline High systolic BP & $1.26(1.14$ to 1.40$)$ & 1.24 (1.11 to 1.39$)$ & 1.20 (1.08 to 1.32$)$ & $1.20(1.08$ to 1.31$)$ & 0.78 \\
\hline High diastolic BP & 1.09 (0.81 to 1.46$)$ & 1.14 (0.82 to 1.58$)$ & $0.92(0.74$ to 1.15$)$ & $1.06(0.82$ to 1.35$)$ & 0.10 \\
\hline High LDLC & 1.24 (1.09 to 1.42$)$ & 1.19 (1.03 to 1.38$)$ & $1.33(1.09$ to 1.62$)$ & 1.31 (1.06 to 1.62$)$ & 0.32 \\
\hline High triglycerides & $1.23(0.90$ to 1.68$)$ & $1.26(0.91$ to 1.76$)$ & 1.93 (1.53 to 2.44$)$ & $1.92(1.45$ to 2.54$)$ & 0.49 \\
\hline Low HDLc & 1.35 (1.18 to 1.55$)$ & $1.23(1.06$ to 1.43$)$ & $1.41(1.25$ to 1.60$)$ & $1.36(1.20$ to 1.54$)$ & 1.00 \\
\hline High glucose & 1.05 (0.85 to 1.30$)$ & $1.02(0.82$ to 1.27$)$ & 1.24 (1.08 to 1.42$)$ & 1.20 (1.03 to 1.40$)$ & 0.002 \\
\hline High insulin & 1.58 (1.33 to 1.87$)$ & $1.48(1.22$ to 1.79$)$ & 1.99 (1.72 to 2.30$)$ & 1.95 (1.65 to 2.29$)$ & $<0.001$ \\
\hline
\end{tabular}

BMI=body mass index; LDLc=low density lipoprotein cholesterol; HDLc=high density lipoprotein cholesterol.

*High systolic blood pressure $\geq 130 \mathrm{~mm} \mathrm{Hg}$; high diastolic blood pressure $\geq 85 \mathrm{~mm} \mathrm{Hg}$; high LDLc $\geq 2.75 \mathrm{mmol} / \mathrm{l}$; high triglycerides $\geq 1.7 \mathrm{mmol} / \mathrm{l}$; low HDLC $<1.03 \mathrm{mmol} / \mathrm{l}$; high glucose $\geq 5.6 \mathrm{mmol} / \mathrm{l}$; high insulin $\geq 16.95 \mathrm{IU} / \mathrm{l}$.

†Adjusted for age, height, and height ${ }^{2}$.

ҒAs for model 1 plus adjusted for maternal age, parity, family social class, maternal education, paternal education, birth weight, gestational age, maternal and paternal BMI, and puberty (additional adjustment for age at menarche in girls did not alter associations presented here for girls).

§Interaction test for association in fully adjusted model (model 2): tests null hypothesis that fully adjusted associations in girls and boys are same.

increases in risk in those at the upper end of the distribution (see figs 1a-1j on bmj.com).

Table 3 shows the association of change in adiposity z scores between 9-12 years and 15-16 years with adolescent cardiovascular risk factors as binary outcomes at age 15-16. In both sexes a greater increase in adiposity SD score between the two ages was associated with increased odds of adverse levels of systolic blood pressure and adverse concentrations of fasting low density lipoprotein cholesterol, triglycerides, high density lipoprotein cholesterol, and insulin for all three measures of adiposity. In boys only a greater increase in adiposity $\mathrm{z}$ scores between these ages was also associated with an increased odds of high glucose concentrations.

Table 4 shows the association between change in overweight/obesity status according to the International Obesity Task Force and risk factors as binary outcomes. Consistent with results in tables 1-3 there were no associations between these categories and diastolic blood pressure (data not shown). Girls who were overweight/obese at age 9-12 but were normal weight by 15-16 had similar odds of adverse levels of risk factors to those who were normal weight at both ages. In boys odds of high systolic blood pressure, high concentrations of triglycerides and insulin, and low concentrations of high density lipoprotein cholesterol remained higher in this group than in those who were normal weight at both ages, but were lower than in those who remained overweight/obese at both ages or who were normal at age 9-12 and then overweight/obese at age 15-16.

Tables I and J on bmj.com show characteristics of the cohort by categories of overweight/obesity. In both boys and girls, those who were overweight/obese at age $9-12$ but normal weight by $15-16$ had lower mean BMI, waist circumference, and fat mass at the 9-10 and 11-12 assessments than those who were overweight/ obese at age 9-12 and remained in this category at 1516. By contrast mean systolic blood pressure at the 9-10 and 11-12 assessments was similar in those who changed from overweight/obese to normal compared with those who remained overweight/obese at both ages. In terms of parental characteristics that might distinguish the children who were overweight/obese at 9-12 but became normal weight by 15-16 from those who remained overweight/obese at both ages, mother's BMI before pregnancy was on average lower, they 
Table 2 | Prospective associations between BMI, waist, and fat mass (assessed at age 9-12) and cardiovascular risk factor as continuously measured outcomes at age 15-16 in all eligible participants with missing data imputed by multivariate multiple imputation ( $n=5235$ ). Figures are mean differences (null value 0 ) or ratios of geometric means (for triglycerides and insulin, null value 1) of outcome per 1SD (z score) of exposure ( $95 \%$ confidence interval)

\begin{tabular}{|c|c|c|c|c|c|}
\hline \multirow{2}{*}{$\begin{array}{l}\text { Exposure and } \\
\text { outcome }\end{array}$} & \multicolumn{2}{|c|}{ Girls $(n=2747)$} & \multicolumn{2}{|c|}{ Boys $(n=2488)$} & \multirow{2}{*}{$\begin{array}{c}\text { P value for sex } \\
\text { interaction } \ddagger\end{array}$} \\
\hline & Model 1* & Model $2 \dagger$ & Model 1* & Model $2 \dagger$ & \\
\hline \multicolumn{6}{|l|}{ BMI } \\
\hline Systolic BP (mm Hg) & 1.54 (1.12 to 1.96$)$ & 1.51 (1.07 to 1.98$)$ & $1.28(0.86$ to 1.71$)$ & 1.09 (0.67 to 1.52$)$ & 0.18 \\
\hline Diastolic BP (mm Hg) & $-0.09(-0.42$ to 0.25$)$ & $-0.05(-0.37$ to 0.31$)$ & $-0.15(-0.53$ to 0.23$)$ & $-0.03(-0.40$ to 0.44$)$ & 0.94 \\
\hline $\operatorname{LDLc}(\mathrm{mmol} / \mathrm{l})$ & 0.06 (0.03 to 0.09$)$ & 0.04 (0.02 to 0.07$)$ & 0.06 (0.04 to 0.09$)$ & 0.07 (0.05 to 0.10$)$ & 0.10 \\
\hline HDLc (mmol/l) & $-0.04(-0.06$ to -0.02$)$ & $-0.04(-0.06$ to -0.02$)$ & $-0.05(-0.07$ to -0.03$)$ & $-0.05(-0.07$ to -0.03$)$ & 0.49 \\
\hline Glucose (mmol/l) & $0.00(-0.03$ to 0.03$)$ & $0.01(-0.02$ to 0.04$)$ & 0.07 (0.05 to 0.08$)$ & 0.06 (0.05 to 0.07$)$ & $<0.001$ \\
\hline Triglycerides§ & $1.03(1.00$ to 1.05$)$ & 1.04 (1.01 to 1.05$)$ & 1.07 (1.04 to 1.08$)$ & 1.07 (1.04 to 1.08$)$ & 0.03 \\
\hline Insulin§ & 1.09 (1.07 to 1.12$)$ & 1.08 (1.06 to 1.10$)$ & $1.22(1.19$ to 1.23$)$ & 1.20 (1.18 to 1.21$)$ & $<0.001$ \\
\hline \multicolumn{6}{|l|}{ Waist circumference } \\
\hline Systolic BP (mm Hg) & 1.45 (0.98 to 1.92$)$ & $1.33(0.82$ to 1.84$)$ & 1.15 (0.66 to 1.62$)$ & $0.89(0.41$ to 1.38$)$ & 0.22 \\
\hline Diastolic BP (mm Hg) & $-0.24(-0.61$ to 0.12$)$ & $-0.20(-0.58$ to 0.18$)$ & $-0.48(-0.89$ to -0.07$)$ & $-0.27(-0.69$ to 0.13$)$ & 0.81 \\
\hline $\operatorname{LDLc}(\mathrm{mmol} / \mathrm{l})$ & 0.07 (0.05 to 0.09$)$ & 0.05 (0.03 to 0.08$)$ & 0.09 (0.06 to 0.11$)$ & 0.08 (0.05 to 0.10$)$ & 0.10 \\
\hline $\mathrm{HDLC}(\mathrm{mmol} / \mathrm{l})$ & $-0.05(-0.07$ to -0.03$)$ & $-0.05(-0.07$ to -0.02$)$ & $-0.06(-0.08$ to -0.03$)$ & $-0.05(-0.08$ to -0.02$)$ & 0.99 \\
\hline Glucose (mmol/l) & $0.00(-0.03$ to 0.03$)$ & $0.01(-0.02$ to 0.04$)$ & 0.06 (0.05 to 0.07$)$ & 0.05 (0.04 to 0.06$)$ & 0.008 \\
\hline Triglycerides & $1.03(1.00$ to 1.05$)$ & 1.04 (1.01 to 1.06$)$ & 1.09 (1.07 to 1.12$)$ & 1.09 (1.07 to 1.12$)$ & 0.003 \\
\hline Insulin & 1.09 (1.07 to 1.12$)$ & 1.08 (1.06 to 1.10$)$ & $1.24(1.22$ to 1.27$)$ & $1.22(1.19$ to 1.24$)$ & $<0.001$ \\
\hline \multicolumn{6}{|l|}{ Fat mass } \\
\hline Systolic BP (mm Hg) & 1.65 (1.19 to 2.12 ) & 1.58 (1.09 to 2.06$)$ & $1.20(0.72$ to 1.65$)$ & $0.92(0.45$ to 1.36$)$ & 0.10 \\
\hline Diastolic BP (mm Hg) & $0.07(-0.29$ to 0.44$)$ & $0.15(-0.24$ to 0.53$)$ & $-0.18(-0.59$ to 0.23$)$ & $-0.10(-0.50$ to 0.30$)$ & 0.38 \\
\hline LDLc (mmol/l) & $0.06(0.03$ to 0.08$)$ & 0.04 (0.02 to 0.07$)$ & 0.06 (0.03 to 0.09$)$ & 0.06 (0.03 to 0.09$)$ & 0.32 \\
\hline $\mathrm{HDLC}(\mathrm{mmol} / \mathrm{l})$ & $-0.04(-0.06$ to -0.02$)$ & $-0.03(-0.05$ to -0.01$)$ & $-0.05(-0.07$ to -0.03$)$ & $-0.04(-0.06$ to -0.02$)$ & 0.49 \\
\hline Glucose (mmol/l) & $0.00(-0.03$ to 0.03$)$ & $0.00(-0.03$ to 0.03$)$ & 0.07 (0.05 to 0.08$)$ & $0.06(0.05$ to 0.07$)$ & $<0.001$ \\
\hline Triglycerides§ & $1.02(0.99$ to 1.04$)$ & $1.02(1.00$ to 1.04$)$ & 1.07 (1.05 to 1.09$)$ & 1.07 (1.05 to 1.09 ) & 0.002 \\
\hline Insulin§ & 1.10 (1.08 to 1.12$)$ & 1.08 (1.06 to 1.10$)$ & 1.22 (1.19 to 1.24$)$ & 1.19 (1.17 to 1.21$)$ & $<0.001$ \\
\hline \multicolumn{6}{|c|}{$\begin{array}{l}\text { BMI=body mass index; LDLC=low density lipoprotein cholesterol; HDLC=high density lipoprotein cholesterol. } \\
\text { *Adjusted for age, height, and height2. } \\
\text { †As for model } 1 \text { plus adjusted for maternal age, parity, family social class, maternal education, paternal education, birth weight, gestational age, } \\
\text { maternal and paternal BMI, and puberty (additional adjustment for age at menarche in girls did not alter associations presented here for girls). } \\
\text { łInteraction test for association in fully adjusted model (model } 2 \text { ): tests null hypothesis that fully adjusted association in girls and boys are } \\
\text { same.§Results are ratios of geometric means for each outcome per } 1 \text { SD greater exposure (BMI, waist, fat mass). }\end{array}$} \\
\hline
\end{tabular}

were less likely to have had three or more previous pregnancies, and were more likely to have a university degree, and both parents were less likely to smoke. Differences in these family characteristics (as measured here), however, could not explain the reduced cardiovascular risk in those who favourably altered their adiposity profile between 9-12 and 15-16 years as all results in table 4 are adjusted for these characteristics.

When we further adjusted the associations between change in overweight/obese categories for baseline BMI (or waist circumference or fat mass) as a continuous variable and also for baseline systolic blood pressure the results were somewhat attenuated compared with those in table 4 , but overall patterns were not different. These adjustments did not explain the differences between those who changed from overweight/ obese at 9-12 to normal at 15-16 compared with those who were overweight/obese at both ages. For example, for girls the odds ratio for high concentration of fasting insulin with these additional adjustments was 0.82 (0.41 to 1.65$)$ in those who were overweight/ obese at age 9-12 and then normal at 15-16;2.85 (1.67 to 4.87 ) in those who were normal at 9-12 and overweight/obese at 15-16; and 1.85 (1.02 to 3.36) in those who were overweight/obese at both ages compared with girls who were normal weight at both ages. The corresponding figures for boys were 1.99 (1.00 to 4.00$), 4.08$ (1.97 to 8.43), and 6.52 (3.56 to 11.81), respectively. When all of these analyses of change were repeated with categories of overweight/ obese defined by thresholds of waist circumference or fat mass they did not differ in their patterns, or substantively in their magnitudes, to those presented here for categories defined by BMI (results available from authors on request).

In models including only potential confounders (but not any of the adiposity measures) between $1 \%$ (for fasting low density lipoprotein cholesterol, triglycerides, and glucose) and 2.5\% (systolic blood pressure, high density lipoprotein cholesterol, insulin) of the variation in cardiovascular outcomes were explained. Addition of BMI only to these models increased the variation explained in the outcome to between $4 \%$ (low density lipoprotein cholesterol, triglycerides, glucose (in boys)) and 7\% (systolic blood pressure and insulin). Further addition of waist circumference or 
Table 3|Prospective associations of change in BMI, waist circumference, and fat mass between age 9-12 and age 15-16 with cardiovascular risk factor as binary outcomes assessed at age 15-16 in all eligible participants with missing data imputed by multivariate multiple imputation $(n=5235)$. Figures are odds ratios of outcome per 1SD ( $z$-score) change of exposure $(95 \%$ confidence interval)

\begin{tabular}{|c|c|c|c|c|c|}
\hline \multirow[b]{2}{*}{ Exposure and outcome* } & \multicolumn{2}{|c|}{ Girls $(n=2747)$} & \multicolumn{2}{|c|}{ Boys $(n=2488)$} & \multirow{2}{*}{$\begin{array}{l}\text { P value for } s \\
\text { interaction }\end{array}$} \\
\hline & Model $1 \dagger$ & Model $2 \ddagger$ & Model $1 \dagger$ & Model $2 \ddagger$ & \\
\hline \multicolumn{6}{|l|}{ Change in BMI z score } \\
\hline High systolic BP & 1.49 (1.28 to 1.73$)$ & 1.52 (1.30 to 1.78$)$ & 1.51 (1.31 to 1.73$)$ & 1.50 (1.30 to 1.73$)$ & 0.98 \\
\hline High diastolic BP & 1.22 (0.81 to 1.85$)$ & 1.35 (0.86 to 2.12$)$ & 1.04 (0.75 to 1.46$)$ & 1.07 (0.76 to 1.51$)$ & 0.57 \\
\hline High LDLc & 1.44 (1.19 to 1.74$)$ & $1.40(1.14$ to 1.71$)$ & 1.80 (1.38 to 2.34$)$ & $1.80(1.38$ to 2.34$)$ & 0.43 \\
\hline High triglycerides & 1.94 (1.33 to 2.83$)$ & 2.23 (1.46 to 3.40$)$ & 2.90 (2.04 to 4.13$)$ & $3.11(2.12$ to 4.57$)$ & 0.27 \\
\hline Low HDLC & $1.82(1.49$ to 2.23$)$ & $1.76(1.42$ to 2.19$)$ & 1.67 (1.36 to 2.05$)$ & 1.67 (1.34 to 2.05$)$ & 0.82 \\
\hline High glucose & 1.06 (0.81 to 1.38$)$ & 1.03 (0.79 to 1.34$)$ & 1.39 (1.16 to 1.67$)$ & 1.38 (1.15 to 1.66$)$ & 0.06 \\
\hline High insulin & 2.24 (1.81 to 2.79$)$ & 2.27 (1.79 to 2.87 ) & 3.63 (2.77 to 4.76$)$ & 3.74 (2.83 to 4.96$)$ & 0.01 \\
\hline \multicolumn{6}{|c|}{ Change in waist circumference $z$ score } \\
\hline High systolic BP & 1.32 (1.15 to 1.52$)$ & 1.35 (1.17 to 1.56$)$ & 1.34 (1.17 to 1.54$)$ & 1.34 (1.17 to 1.54$)$ & 0.99 \\
\hline High diastolic BP & 1.09 (0.76 to 1.57$)$ & 1.17 (0.79 to 1.74$)$ & 1.13 (0.83 to 1.54$)$ & 1.17 (0.85 to 160$)$ & 0.99 \\
\hline High LDLC & 1.36 (1.13 to 1.62$)$ & 1.31 (1.09 to 1.59$)$ & 1.61 (1.24 to 2.10$)$ & $1.63(1.28$ to 2.09$)$ & 0.46 \\
\hline High triglycerides & 1.90 (1.27 to 2.83$)$ & 2.08 (1.34 to 3.21$)$ & 2.44 (1.63 to 3.66$)$ & $2.62(1.83$ to 3.76$)$ & 0.43 \\
\hline Low HDLC & 1.70 (1.39 to 2.08$)$ & 1.67 (1.36 to 2.04$)$ & 1.51 (1.23 to 1.86$)$ & $1.51(1.23$ to 1.86$)$ & 0.69 \\
\hline High glucose & 1.04 (0.81 to 1.33$)$ & 1.01 (0.79 to 1.30$)$ & $1.36(1.16$ to 1.61$)$ & 1.35 (1.14 to 1.60$)$ & 0.05 \\
\hline High insulin & 1.86 (1.51 to 2.28$)$ & 1.85 (1.50 to 2.80$)$ & $3.27(2.48$ to 4.30$)$ & 3.44 (2.67 to 4.43$)$ & 0.004 \\
\hline \multicolumn{6}{|l|}{ Change in fat mass $z$ score } \\
\hline High systolic BP & $1.44(1.25$ to 1.66$)$ & 1.49 (1.28 to 1.74$)$ & 1.24 (1.09 to 1.41$)$ & 1.24 (1.09 to 1.42$)$ & 0.68 \\
\hline High diastolic BP & 1.21 (0.82 to 1.79$)$ & 1.37 (0.90 to 2.09$)$ & 0.81 (0.58 to 1.13$)$ & 0.83 (0.59 to 1.16$)$ & 0.49 \\
\hline High LDLC & 1.45 (1.20 to 1.75$)$ & 1.39 (1.14 to 1.71$)$ & 1.78 (1.44 to 2.22 ) & 1.79 (1.43 to 2.24$)$ & 0.61 \\
\hline High triglycerides & 2.11 (1.45 to 3.07 ) & 2.43 (1.58 to 3.72$)$ & 2.39 (1.76 to 3.25$)$ & 2.46 (1.76 to 3.45$)$ & 0.97 \\
\hline Low HDLC & $1.82(1.50$ to 2.21$)$ & 1.76 (1.42 to 2.18 ) & $1.46(1.22$ to 1.75$)$ & 1.44 (1.21 to 1.72$)$ & 0.84 \\
\hline High glucose & 1.16 (0.88 to 1.51$)$ & 1.12 (0.85 to 1.49$)$ & 1.49 (1.24 to 1.78$)$ & 1.48 (1.24 to 1.77$)$ & 0.09 \\
\hline High insulin & 2.28 (1.84 to 2.81$)$ & 2.33 (1.85 to 2.94$)$ & 3.37 (2.61 to 4.36$)$ & 3.51 (2.69 to 4.57$)$ & 0.03 \\
\hline
\end{tabular}

$\mathrm{BMI}=$ body mass index; LDLc=low density lipoprotein cholesterol; HDLc=high density lipoprotein cholesterol.

*High systolic blood pressure $\geq 130 \mathrm{~mm} \mathrm{Hg}$; high diastolic blood pressure $\geq 85 \mathrm{~mm} \mathrm{Hg}$; high LDLc $\geq 2.75 \mathrm{mmol} / \mathrm{l}$; high triglycerides $\geq 1.7 \mathrm{mmol} / \mathrm{l}$; low HDLc $<1.03 \mathrm{mmol} / \mathrm{l}$; high glucose $\geq 5.6 \mathrm{mmol} / \mathrm{l}$; high insulin $\geq 16.95 \mathrm{IU} / \mathrm{l}$.

†Adjusted for age, height, height ${ }^{2}$, BMI, waist circumference, or fat mass (as appropriate for their respective change exposure) at baseline.

$\ddagger$ As for model 1 plus adjusted for maternal age, parity, family social class, maternal education, paternal education, birth weight, gestational age, maternal and paternal BMI, and puberty (additional adjustment for age at menarche in girls did not alter associations presented here for girls).

§Interaction test for association in fully adjusted model (model 2): tests null hypothesis that fully adjusted association in girls and boys are same.

fat mass did not increase the amount of variation in the outcomes explained by the independent variables. In models including just one of the three measurements of adiposity there were no problems with variance inflation (all variance inflation factors between 1.0 and 1.5). In models with two of the measurements variance inflation factors increased to between 8.0 and 10.5 and with all three in the model they were between 9.5 to 11.5 , suggesting potential problems with collinearity.

When we repeated all analyses limiting the sample to include participants with no missing data (tables K-O on bmj.com), results did not differ substantially from those presented here.

\section{DISCUSSION}

Main findings

Childhood BMI alone adequately identifies those who will be at increased risk of adverse cardiovascular profiles in adolescence, and a direct assessment of fat mass or a measure of central adiposity (waist circumference) is not more strongly associated with adverse outcomes. Children who change from overweight to normal weight improve their cardiovascular profiles compared with those children who remain overweight in childhood and adolescence.

All three measures of adiposity assessed in childhood are prospectively associated with adverse cardiovascular risk factors in adolescents, with similar magnitudes of association. These associations are robust to adjustment for a wide range of potential confounding factors. The magnitude of associations between adiposity in childhood and cardiovascular risk factors assessed in adolescence are similar to equivalent associations found in studies of middle aged adults, ${ }^{23}$ suggesting that greater adiposity begins to adversely influence cardiovascular risk factors even in childhood/adolescence. BMI, waist circumference, and fat mass were all strongly correlated with each other, and neither childhood waist circumference nor fat mass was more strongly associated than BMI with cardiovascular risk factors. The addition of fat mass or waist circumference to multivariable models including BMI did not increase the amount of variation in cardiovascular outcomes explained by the independent variables in models with more than one of these adiposity measures, though there was some evidence of collinearity. 
Table 4 | Multivariable associations of change in BMI category between age 9-12 and 15-16 years with cardiovascular risk factors as binary outcomes in all eligible participants with missing data multiply imputed

\begin{tabular}{|c|c|c|c|c|c|c|c|c|}
\hline \multirow[b]{2}{*}{$\begin{array}{l}\text { BMI category at age } 9-12 \\
\text { and age } 15-16\end{array}$} & \multirow[b]{2}{*}{$\%(\mathrm{SE})^{\star}$} & \multirow[b]{2}{*}{$\begin{array}{l}\text { Mean (SE) BMI } \\
\text { at age } 15\end{array}$} & \multicolumn{6}{|c|}{ Odds ratio $(95 \% \mathrm{Cl})$} \\
\hline & & & $\begin{array}{c}\text { High SBP } \\
(\geq 130 \mathrm{~mm} \mathrm{Hg})\end{array}$ & $\begin{array}{c}\text { High LDLc } \\
(\geq 2.75 \mathrm{mmol} / \mathrm{l})\end{array}$ & $\begin{array}{l}\text { High triglycerides } \\
(\geq 1.7 \mathrm{mmol} / \mathrm{l})\end{array}$ & $\begin{array}{c}\text { Low HDLc } \\
(\ll 1.03 \mathrm{mmol} / \mathrm{l})\end{array}$ & $\begin{array}{l}\text { High glucose } \\
(\geq 5.6 \mathrm{mmol} / \mathrm{l})\end{array}$ & $\begin{array}{l}\text { High insulin } \\
(\geq 16.95 \mathrm{IU} / \mathrm{l})\end{array}$ \\
\hline \multicolumn{9}{|l|}{ Girls $(n=2747)$} \\
\hline Normal at both & $70.7(0.9)$ & $20.2(0.05)$ & Reference & Reference & Reference & Reference & Reference & Reference \\
\hline Overweight-obese/normal & $11.1(0.6)$ & $22.9(0.15)$ & $1.06(0.77$ to 1.47$)$ & $1.04(0.67$ to 1.61$)$ & 1.81 (0.72 to 4.52$)$ & 1.01 (0.65 to 1.58$)$ & 0.83 (0.47 to 1.47$)$ & 0.97 (0.55 to 1.73$)$ \\
\hline Normal/overweight-obese & $4.8(0.4)$ & $25.5(0.11)$ & 1.61 (1.04 to 2.47 ) & 1.55 (0.91 to 2.66$)$ & 4.41 (1.73 to 11.22$)$ & 2.25 (1.34 to 3.79$)$ & $0.86(0.36$ to 2.06$)$ & 3.37 (1.20 to 1.42$)$ \\
\hline Overweight-obese at both & $13.4(0.6)$ & $28.1(0.18)$ & $1.74(1.29$ to 2.33$)$ & $1.59(1.07$ to 2.36$)$ & 2.88 (1.20 to 6.89$)$ & $2.30(1.50$ to 3.54$)$ & 1.05 (0.55 to 1.99$)$ & 2.87 (1.77 to 4.66$)$ \\
\hline \multicolumn{9}{|l|}{ Boys $(n=2488)$} \\
\hline Normal at both & $74.6(0.8)$ & $19.6(0.04)$ & Reference & Reference & Reference & Reference & Reference & Reference \\
\hline Overweight-obese/normal & $9.1(0.6)$ & $22.5(0.15)$ & $1.60(1.20$ to 2.14$)$ & $1.37(0.75$ to 2.49$)$ & $3.35(1.42$ to 7.91$)$ & $1.50(1.00$ to 2.11$)$ & $1.18(0.77$ to 1.79$)$ & $2.43(1.33$ to 4.45$)$ \\
\hline Normal/overweight-obese & $4.1(0.4)$ & $24.5(0.10)$ & $1.58(1.03$ to 2.41$)$ & $2.35(1.14$ to 4.85$)$ & $5.38(1.97$ to 14.65$)$ & 2.34 (1.43 to 3.83$)$ & $1.25(0.71$ to 2.20$)$ & $4.35(2.13$ to 8.91$)$ \\
\hline Overweight-obese at both & $12.2(0.7)$ & $27.3(0.18)$ & $1.92(1.49$ to 2.47$)$ & $2.29(1.40$ to 3.77$)$ & 8.04 (4.06 to 15.93$)$ & 2.81 (2.00 to 3.96$)$ & 1.81 (1.24 to 2.65$)$ & $8.48(5.47$ to 13.15$)$ \\
\hline \multicolumn{9}{|c|}{$\begin{array}{l}\text { BMI=body mass index; LDLc=low density lipoprotein cholesterol; HDLc=high density lipoprotein cholesterol. } \\
\text { *Percentage (\%) and standard error (SE) in each category across the datasets provides as results from multivariate multiple imputation analyses are based on combining results across } 20 \\
\text { databases so exact numbers in each category cannot be provided. } \\
\text { †Adjusted for age, height, height }{ }^{2} \text {, maternal age, parity, family social class, maternal education, paternal education, birth weight, gestational age, maternal and paternal BMI, and puberty } \\
\text { (additional adjustment for age at menarche in girls did not alter associations). }\end{array}$} \\
\hline
\end{tabular}

Girls who were overweight/obese at age 9-12 but were normal weight by $15-16$ had similar odds of adverse levels of risk factors to those who were normal weight at both ages. In boys odds of higher systolic blood pressure and higher concentrations of triglycerides and insulin and lower concentrations of high density lipoprotein cholesterol remained higher in this group compared with those who were normal weight at both ages but were lower than in those who remained overweight/obese at both ages or who were normal at age 9-12 and then overweight/obese at age 15-16. Thus, in both sexes changing from overweight/ obese at age 9-12 to normal weight at age 15-16 was associated with better risk profiles than remaining overweight/obese from childhood through to adolescence. Those who made this change to normal weight had on average lower mean levels of BMI, waist circumference, and fat mass at age 9-12 than those who remained overweight/obese at both ages and also had somewhat lower systolic blood pressure. When we adjusted for these baseline characteristics, however, the improvements in those moving to the normal category remained.

\section{Comparisons with other studies and implications}

Few previous studies have examined change in adiposity between childhood and adolescence/early adulthood in relation to cardiovascular risk factors. Of those that have, all suggest that those whose adiposity decreases (relative to age and sex standards) have improved cardiovascular risk profiles, whereas those whose levels increase tend to have worse profiles. ${ }^{4524}$ Contrary to concerns about BMI not being a suitable measurement of adiposity in childhood, ${ }^{13}{ }^{14}$ we found no difference in the magnitudes of prospective associations between BMI, waist circumference, or fat mass in the whole cohort or in analyses restricted to those in the normal BMI range, suggesting that with respect to identifying those with more adverse cardiovascular risk profiles BMI is just as suitable as waist circumference or fat mass in children. This work builds on a previous publication from the Avon Longitudinal Study in which the cross sectional associations between blood pressure in childhood and BMI and fat mass determined by dual energy $\mathrm{x}$ ray absorptiometry were compared and found to be similar. ${ }^{25}$ That study did not consider waist circumference, nor outcomes other than blood pressure, and the study was cross sectional. It also builds on a cross sectional study of data at age 9-10, which found similar magnitudes of association between BMI, waist, and fat mass and cardiovascular risk factors. ${ }^{26}$ That study was cross sectional and assessed non-fasting lipids and did not have information on glucose or insulin. Waist circumference might be less reliably measured than BMI or fat mass and were it possible to measure it more reliably it might be associated more strongly with cardiovascular risk factors. Recent evidence suggests that where waist circumference is measured in overweight children affects its relation with cardiovascular risk factors, with the mid-point between the ribs and iliac crest (as used here) or the narrowest circumference being more strongly associated with risk factors than the circumference at the level of the umbilicus or the iliac crest. ${ }^{27}$ Thus, it is unlikely that our results are affected by measuring waist circumference at an inappropriate site. Marked central distribution of adiposity might not occur until after puberty, and it will be worth repeating our analyses as these children go into adulthood. Recent findings in adults, however, suggest that BMI and waist circumference are similarly associated with cardiovascular disease events, ${ }^{28}$ suggesting that general adiposity is as useful as its distribution for identifying those at risk or that better measurements (that is, better than waist circumference) are required for identifying detrimental central adiposity, in particular visceral adiposity.

One implication of our findings is that BMI in children is suitable for identifying those at risk of future adverse cardiovascular risk profiles in clinical practice 
and public health surveillance and that in these settings use of more sophisticated measurements of fat mass and adiposity distribution are unlikely to be needed. In research projects, particularly in large epidemiological studies where the addition of more sophisticated measurements might increase costs markedly, these results could suggest that fat mass determined by dual energy $x$ ray absorptiometry and waist circumference are unnecessary. We would argue against this for several reasons. Firstly, while our results show similar associations with cardiovascular risk factors we have previously reported differences with determinants or risk factors for greater adiposity in this cohort. For example, we found a stronger association between socioeconomic position and fat mass than with $\mathrm{BMI}^{29}$ and that the FTO genotype was specifically associated with fat mass. ${ }^{30}$ Secondly, it would be valuable to see if our findings can be replicated in other cohort studies, including those with different ethnic groups in whom sensitivity to metabolic effects of adiposity can be much greater. Thirdly, as noted above, as the participants in this cohort age the correlation between the three measurements of adiposity might reduce and differences in how they relate to risk factors might emerge.

It is unclear why we found no prospective association between adiposity and diastolic blood pressure. Other early life risk factors, including birth weight, gestational age, and socioeconomic position, are associated with systolic blood pressure but not with (or more weakly with) diastolic blood pressure. ${ }^{31}$ Our finding that associations of adiposity measured in childhood (9-12 years) with cardiovascular risk factors in adolescence (15-16) were stronger in boys than girls for fasting insulin, glucose, and triglyceride concentrations has not been previously reported, and in adults adiposity is similarly associated with cardiovascular disease events and risk factors in women and men. ${ }^{23}$ A small study ( $\mathrm{n}=193$-350 for different analyses) with detailed measurements including repeat assessment with the euglycaemic clamp, assessed on three occasions from age 11 to 19 , found that insulin resistance and triglyceride concentrations increased in boys and high density lipoprotein cholesterol concentrations decreased as they moved from childhood through adolescence, despite a decrease in body fat (and increase in lean mass) over this time period. ${ }^{32}$ In girls they found the opposite (that is, an increase in body fatness but a decrease in insulin resistance as they went through adolescence). Thus, our findings might have been influenced by sex differences occurring during the transition through puberty and might be related to where fat is placed - that is, preferentially more subcutaneous and "safe" weight gain in girls than in boys. ${ }^{32}$

The linear associations found across the whole distributions of childhood adiposity suggest that there is no threshold effect of increase in cardiovascular risk at high levels of adiposity in children. This finding highlights the importance of prevention strategies aimed at shifting the population distribution of childhood adiposity downwards. This noted, some children who were overweight/obese age 9-12 seemed to have normalised their weight category status without formal intervention that we are aware of and in doing so improved their cardiovascular profile. The children in this group were on average somewhat less adipose than those who did not move down to the normal weight group but adjustment for mean BMI (or waist circumference or fat mass) at baseline did not markedly alter the associations between change in weight category status and cardiovascular risk factors.

\section{Study strengths and limitations}

We examined associations prospectively, compared three different measurements of adiposity and changes in these over time, and adjusted for a wide range of potential confounding factors. Consistent with all other birth cohorts and prospective cohorts in general there has been loss to follow-up, with those who continue to attend regular follow-up clinics being more likely to be from higher socioeconomic backgrounds. ${ }^{15}$ We cannot, however, think of any reason why adiposity should be differently associated with cardiovascular risk factors in those who were lost to follow-up. Our multivariate multiple imputation analysis suggests that there are no major problems with selection bias between those with no missing data (including on fasting bloods) and the whole eligible cohort who attended the follow-up clinics. Our measurements of adiposity were obtained when nearly all the children will have been prepubertal. Because we preferentially used measurements from the 9-10 clinic, and used those from the 11-12 clinic when these were missing, $75 \%$ of participants were aged under 10 at the time of baseline assessment and $82.5 \%$ were Tanner stage I or II by parental report with just $2.5 \%$ of the girls having gone through menarche. As discussed above, it is possible that measurements of central adiposity after puberty relate more strongly to cardiovascular risk factors. The participants in this study are predominantly of European origin; all were born and most were brought up in the UK. We cannot assume that these findings would apply to other ethnic groups or to children brought up in difference environments.

\section{Conclusion}

In summary, our results show that greater adiposity at age 9-12 is associated with adverse cardiovascular risk factors at age 15-16 and that these associations are continuous across the full range of general population levels of childhood adiposity and robust to adjustment for a wide range of potential confounding factors. BMI in childhood is prospectively associated with cardiovascular risk factors with the similar magnitudes of association as fat mass or waist circumference. It is reassuring that our results suggest that change to normal weight by adolescence among those who are overweight/obese in childhood is associated with better cardiovascular risk profiles than in those who remain overweight. Our findings highlight the need to shift the whole distribution of adiposity in children downwards and to develop interventions that safely and effectively reduce weight and improve cardiovascular risk factors in overweight/obese children. 


\section{WHAT IS ALREADY KNOWN ON THIS TOPIC}

Higher BMI in childhood or adolescence is associated with increased risk of cardiovascular disease in later life

Cross sectional studies in childhood show associations between BMI and cardiovascular risk factors, but there are few prospective studies

BMI is said to be a poor measure of adiposity, particularly in childhood, and the magnitude of its association with risk factors might underestimate the true adverse effect of greater adiposity in this age group

\section{WHAT THIS STUDY ADDS}

BMI, waist circumference, and total fat mass assessed at age 9-12 are positively associated with cardiovascular risk factors at age 15-16

The magnitudes of these associations are similar for all measures of adiposity used

Girls who favourably alter their overweight status between childhood and adolescence have cardiovascular risk profiles broadly similar to those who were normal weight at both time points, but boys who change from overweight to normal show risk factor profiles intermediate between the normal at both ages and overweight at both ages
We thank all the families who took part in this study, the midwives for their help in recruiting them, and the whole ALSPAC team, which includes interviewers, computer and laboratory technicians, clerical workers, research scientists, volunteers, managers, receptionists, and nurses. Contributors: DAL proposed the study objectives, obtained funds for the fasting blood assays, wrote the statistical protocol, undertook analyses, and wrote the first draft of the paper. LB helped with data cleaning and revised later drafts of the paper. JL contributed to developing the study objectives and revised later drafts of the paper. KT contributed to the statistical protocol and revised later drafts of the paper. LDH contributed to the statistical protocol and revised later drafts of the paper. AF contributed to developing the study objectives and revised later drafts of the paper. LC and PW completed assays on fasting blood samples and revised later drafts of the paper. ARN obtained funds for the DXA scans and other anthropometric data, contributed to developing the study objectives, and revised later drafts of the paper. GDS is scientific directo of ALSPAC and has obtained core funding for this study over the past six years, contributed to developing the study objectives, and revised later drafts of the paper. NS supervised the laboratory assays on the fasting blood samples, contributed to developing the study objectives, and revised later drafts of the paper. DAL is guarantor.

Funding: This study was funded by a grant from US National Institutes of Health $(\mathrm{NIH})$ and the National Institute of Diabetes and Digestive and Kidney Diseases (R01 DK077659). The Medical Research Council (MRC) the Wellcome Trust, and the University of Bristol provide core funding support for ALSPAC. The MRC (G0600705) and the University of Bristo provide core funding for the MRC Centre of Causal Analyses in Translational Epidemiology. AF is funded by a UK Medical Research Council research fellowship. The views expressed in this paper are those of the authors and not necessarily those of any funding body or others whose support is acknowledged. The funders had no role in study design, data collection and analysis, decision to publish, or preparation of the manuscript.

Competing interests: All authors have completed the Unified Competing Interest form at www.icmje.org/coi_disclosure.pdf (available on request from the corresponding author) and declare: no support from any institution for the submitted work; no financial relationships with any institutions that might have an interest in the submitted work in the previous 3 years; no other relationships or activities that could appear to have influenced the submitted work.

Ethical approval: This study was approved by the ALSPAC law and ethics committee (IRB 00003312) and the local research ethics committee. Data sharing: All scientists are able and encouraged to use ALSPAC data. Requests for data to conduct research are made via the ALSPAC executive on a form that is available from the study website: www.bristol. ac.uk/alspac/ (please click on the "scientific community" button for this information). Most requests are accepted and anonymised datasets provided. Where a request is made to conduct research that overlaps with another scientist the executive try to facilitate collaboration between the groups if possible. ALSPAC is a large prospective cohort study with repeat collections of data on parents and children over a 17 year period.
There is no single protocol document but full details of the study and each period of data collection are available at the study website: www.bristol. ac.uk/alspac/ (please click on the "scientific Community" button for this information)

1 Baker JL, Olsen LW, Sorensen TI. Childhood body-mass index and the risk of coronary heart disease in adulthood. $N$ Engl J Med 2007;357:2329-37.

2 Bjorge T, Engeland A, Tverdal A, Davey Smith G. Body mass index in adolescence in relation to cause-specific mortality: a follow-up of 230,000 Norwegian adolescents. Am J Epidemiol 2008;168:30-7.

3 Owen CG, Whincup PH, Orfei L, Chou QA, Rudnicka AR, Wathern AK, et al. Is body mass index before middle age related to coronary heart disease risk in later life? Evidence from observational studies. Int J Obes (Lond) 2009;33:866-77

4 Li S, Chen W, Srinivasan SR, Bond MG, Tang R, Urbina EM, et al. Childhood cardiovascular risk factors and carotid vascular changes in adulthood: the Bogalusa Heart Study. JAMA 2003;290:2271-6.

5 Raitakari OT, Juonala M, Kahonen M, Taittonen L, Laitinen T, Maki-Torkko N, et al. Cardiovascular risk factors in childhood and carotid artery intima-media thickness in adulthood: the Cardiovascular Risk in Young Finns Study. JAMA 2003;290:2277-83.

6 Li X, Li S, Ulusoy E, Chen W, Srinivasan SR, Berenson GS. Childhood adiposity as a predictor of cardiac mass in adulthood: the Bogalusa Heart Study. Circulation 2004;110:3488-92.

7 Srinivasan SR, Bao W, Wattigney WA, Berenson GS. Adolescent overweight is associated with adult overweight and related multiple cardiovascular risk factors: the Bogalusa Heart Study. Metabolism 1996;45:235-40.

8 Sinaiko AR, Donahue RP, Jacobs DR Ir, Prineas RJ. Relation of weight and rate of increase in weight during childhood and adolescence to body size, blood pressure, fasting insulin, and lipids in young adults. The Minneapolis Children's Blood Pressure Study. Circulation 1999;99:1471-6.

9 Must A, Strauss RS. Risks and consequences of childhood and adolescent obesity. Int J Obesity 1999;23(suppl):S2-11.

10 Sinha R, Fisch G, Teague B, Tamborlane WV, Banyas B, Allen K, et al. Prevalence of impaired glucose tolerance among children and adolescents with marked obesity. N Engl J Med 2002;346:802-10.

11 Fraser A, Longnecker M.P, Lawlor DA. Prevalence of elevated alanineaminotransferase (ALT) among US adolescents and associated factors: NHANES 1999-2004. Gastroenterology 2007;133:1814-20.

12 Freedman DS, Katzmarzyk PT, Dietz WH, Srinivasan SR, Berenson GS. Relation of body mass index and skinfold thicknesses to cardiovascular disease risk factors in children: the Bogalusa Heart Study. Am / Clin Nutr 2009;90:210-6.

13 Maynard LM, Wisemandle W, Roche AF, Chumlea WC, Guo SS, Siervogel RM. Childhood body composition in relation to body mass index. Pediatrics 2001;107:344-50.

14 Freedman DS, Wang J, Maynard LM, Thornton JC, Mei Z, PiersonJr RN, et al. Relation of BMI to fat and fat-free mass among children and adolescents. Int J Obesity 2005;29:1-8.

15 Golding J, Pembrey M, Jones R. ALSPAC-the Avon Longitudina Study of Parents and Children. I. Study methodology. Paediatr Perinat Epidemiol 2001;15:74-87.

16 Lawlor DA, Timpson N, Harbord RM, Leary S, Ness A, McCarthy MI, et al. Exploring the developmental overnutrition hypothesis using parental-offspring associations and the FTO gene as an instrumental variable for maternal adiposity. The Avon Longitudinal Study of Parents and Children (ALSPAC). PloS Medicine 2008;5:e33.

17 Royston P. Multiple imputation of missing values. Stata 2004;4:227-41.

18 Zimmet P, Alberti G, Kaufman F, Tajima N, Silink M, Arslanian S, et al. The metabolic syndrome in children and adolescents. Lancet 2007;369:2059-61.

19 Cole TJ, Bellizzi MC, Flegal KM, Dietz WH. Establishing a standard definition for child overweight and obesity worldwide: international survey. BMJ 2000;320:1240-3.

20 McCarthy HD, Jarrett KV, Crawley HF. The development of waist circumference percentiles in British children aged 5.0-16.9 y. Eur J Clin Nutr 2001;55:902-7.

21 Efron B, Tibshirani RJ. An introduction to the bootstrap. Chapman and Hall, 1993.

22 Armitage P, Berry G. Statistical methods in medical research. Blackwell Science, 1994.

23 Whitlock G, Lewington S, Sherliker P, Clarke R, Emberson J, Halsey J, et al. Body-mass index and cause-specific mortality in 900000 adults: collaborative analyses of 57 prospective studies. Lancet 2009;373:1083-96

24 Mamun AA, Lawlor DA, O'Callaghan MJ, Williams GW, Najman JM. The effect of body mass index changes between ages 5 and 14 on blood pressure at age 14: findings from a birth cohort. Hypertension 2005;45:1083-7. 
25 Brion MA, Ness AR, Davey Smith G, Leary SD. Association between body composition and blood pressure in a contemporary cohort of 9 year-old children. J Hum Hypertens 2007;21:283-90.

26 Falaschetti E, Hingorani AD, Jones A, Charakida M, Finer N, Whincup P, et al. Adiposity and cardiovascular risk factors in a large contemporary population of pre-pubertal children. Eur Heart J 2010 Oct 23, epub ahead of print.

27 Johnson ST, Kuk JL, Mackenzie KA, Huang T, Rosychuk RJ, Ball GD. Metabolic risk varies according to waist circumference measurement site in overweight boys and girls. J Pediatr 2010;156:247-52.

28 Taylor AE, Ebrahim S, Ben-Shlomo Y, Martin RM, Whincup PH, Yarnell JW, et al. Comparison of the associations of BMI and measures of central adiposity and fat mass with coronary heart disease, diabetes and all cause mortality: a study using data from four UK cohorts. Am J Clin Nutr 2010;91:547-56.
29 Ness AR, Leary S, Reilly J, Wells J, Tobias J, Clark E, et al. The social patterning of fat and lean mass in a contemporary cohort of children. Int J Pediatr Obes 2006;1:59-61.

30 Frayling TM, Timpson NJ, Weedon MN, Zeggini E, Freathy RM, Lindgren CM, et al. A common variant in the FTO gene is associated with body mass index and predisposes to childhood and adult obesity. Science 2007;316:889-94.

31 Lawlor DA, Davey Smith G. Early life determinants of adult blood pressure. Curr Opin Nephrol Hypertens 2005;14:259-64.

32 Moran A, Jacobs DR Jr, Steinberger J, Steffen LM, Pankow JS, Hong CP, et al. Changes in insulin resistance and cardiovascular risk during adolescence: establishment of differential risk in males and females. Circulation 2008;117:2361-8.

Accepted: 14 September 2010 